\title{
A AGENDA 2030 E O CONTEÚDO INFORMACIONAL MEDIADO POR BIBLIOTECAS EM SERVICYOS DE REDES SOCIAIS ONLINE: UM ESTUDO DE CASO PARA A BIBLIOTECA CENTRAL DA UNIVERSIDADE FEDERAL DO PARÁ
}

Kelren Cecília dos Santos Lima da Mota

Possui graduação em Biblioteconomia pela Universidade Federal do Pará. Especialista em gestão de Bibliotecas Escolares pela Unyleya e mestranda em Ciência da Informação pela Universidade Federal do Pará. E-mail: kelrenlima@ufpa.br

Fernando de Assis Rodrigues

Professor Adjunto no Instituto de Ciências Sociais Aplicadas, lotado na Faculdade de Arquivologia e no Programa de PósGraduação em Ciência da Informação da Universidade Federal do Pará. Doutor e Mestre em Ciência da Informação pela UNESP Universidade Estadual Paulista. E-mail:fernando@rodrigues.pro.br

\section{RESUMO}

As Tecnologias de Informação e Comunicação possibilitaram novas formas para o processo de mediação da informação no âmbito das bibliotecas universitárias, disponibilizando ferramentas, como os Serviços de Redes Sociais Online, que proporciona maior interação com o usuário. Os Serviços de Redes Sociais Online estão sendo aplicados por diversos setores da sociedade e apresentam baixo custo de investimento financeiro para um grande alcance perante a sociedade. Neste contexto, esta pesquisa tem como objetivo analisar o conteúdo informacional que está sendo mediado por meio dos perfis da Biblioteca Central Dr. Clodoaldo Beckmann nos Serviços de Redes Sociais Online, mais especificamente o perfil institucional da Biblioteca no Facebook, com o intuito de compreender o seu papel no desenvolvimento social e sustentável da Região Amazônica, com enfoque na relação com a Agenda 2030. Pesquisas demonstram apesar dos avanços, os Indicadores de Desenvolvimento Humano (IDH) na Amazônia ainda são inferiores às médias nacionais. Em 2016, o Programa das Nações Unidas para o Desenvolvimento realizou uma lista classificatória (ranking) de Índices de Desenvolvimento Humano Médio (IDHM) dos municípios brasileiros, onde constatou-se que o município de Melgaço, Estado 
do Pará, apresenta o menor índice da Amazônia Brasileira. Desta forma, a pesquisa verifica se os dados coletados por meio das postagens do perfil da biblioteca no Facebook estão de acordo com o que preconiza as diretrizes estipuladas pela IFLA, na qual denomina que as bibliotecas são agentes promotores do desenvolvimento social e sustentável, por possibilitarem o acesso público à informação. De acordo com os procedimentos metodológicos, esta pesquisa está classificada como estudo de caso, utilizando como técnica a observação sistemática. O instrumento utilizado foi um formulário baseado em estudos prévios e as diretrizes propostas pela IFLA, onde os dados foram categorizados quanto ao tipo de conteúdo e as características da mensagem. A amostra foi delimitada as postagens realizadas no período dos meses de março a setembro do ano de 2019. Conclui que a Biblioteca Central Dr. Clodoaldo Beckmann ainda prioriza mediar informações quanto a divulgação de seus produtos e serviços. No entanto, percebe-se uma preocupação desta em mediar informações que possam contribuir para o bem-estar e saúde e também, para a elevação do nível de aprendizagem dos estudantes, sendo este tipo de conteúdo classificado como informações gerais, mediado através do quadro Dicas da Semana, que divulga informações quanto ao incentivo à leitura, gerenciamento do tempo de estudo, preservação do acervo, acessibilidade e técnicas de memorização. Por ser uma das maiores bibliotecas universitárias da Região Norte do Brasil, outro fator utilizado para ampliar o acesso a informação para o desenvolvimento humano e social da Região Amazônica são as postagens do tipo Campanha, que são realizadas em parceria com instituições tais como: a Comunidade Cruzeirinho do baixo Acará, o Hospital Universitário João de Barros Barreto, a Superintendência do Sistema Prisional do Estado do Pará, a Fundação Centro de Hematologia e Hemoterapia do Pará. Contribuindo para o alcance dos objetivos da Agenda 2030 que visa garantir que todos os seres humanos possam desenvolver o seu potencial quanto a dignidade e igualdade, em um ambiente saudável e acessível a todos.

PALAVRAS-CHAVE: Bibliotecas universitárias. Desenvolvimento Sustentável. Desenvolvimento Social. Amazônia.

\title{
THE 2030 AGENDA AND THE INFORMATIONAL CONTENT MEDIATED BY LIBRARIES AT ONLINE SOCIAL NETWORK SERVICES: A CASE OF STUDY TO FEDERAL UNIVERSITY OF PARÁ'S CENTRAL LIBRARY
}

\begin{abstract}
Information and Communication Technologies have enabled new ways for the information mediation process within university libraries, providing tools such as Online Social Networking Services, which provides greater interaction with the user. Online Social Networking Services are being applied by various sectors
\end{abstract}


of society and have low cost of financial investment to reach society. In this context, this research aims to analyze the informational content that is being mediated through the profiles of the Dr. Clodoaldo Beckmann Central Library in the Online Social Networking Services, more specifically the institutional profile of the Facebook Library, in order to understand the their role in the social and sustainable development of the Amazon Region, focusing on the relationship with the 2030 Agenda. Research shows despite advances, the Human Development Indicators (HDI) in the Amazon are still below national averages. In 2016, the United Nations Development Program conducted a ranking list (ranking) of Medium Human Development Indexes (MHDI) of Brazilian municipalities, where it was found that the municipality of Melgaço, State of Pará, has the lowest Brazilian Amazon. In this way, the research verifies if the data collected through the posts of the library profile on Facebook are in accordance with the guidelines established by IFLA, which states that libraries are agents promoting social and sustainable development, as they enable public access to information. According to the methodological procedures, this research is classified as a case study, using systematic observation as a technique. The instrument used was a form based on previous studies and the guidelines proposed by IFLA, where the data were categorized according to the type of content and the characteristics of the message. The sample was delimited the posts made from March to September of 2019. It concludes that the Dr. Clodoaldo Beckmann Central Library still prioritizes mediating information about the dissemination of its products and services. However, there is a concern of this in mediating information that can contribute to well-being and health, and also, to raise the level of learning of students, and this type of content classified as general information, mediated through the Tips of the Week, which disseminates information on reading incentives, time management, preservation of collections, accessibility and memorization techniques. As one of the largest university libraries in the Northern Region of Brazil, another factor used to expand access to information for the human and social development of the Amazon Region are Campaign-type posts, which are made in partnership with institutions such as: Cruzeirinho do Baixo Acará, the João de Barros Barreto University Hospital, the Superintendence of the Pará State Prison System, the Pará Hematology and Hemotherapy Center Foundation. develop their potential for dignity and equality in a healthy environment accessible to all.

KEYWORDS: University libraries. Sustainable development. Social development. Amazon.

\section{INTRODUÇÃO}

As Tecnologias de Informação e Comunicação (TIC) possibilitam novas formas para o processo de mediação da informação no âmbito das bibliotecas universitárias. Hoje, as bibliotecas possuem maior interação com os seus usuários, intensificada a partir da Web 2.0. De acordo com Aguiar (2012), a Web 2.0 representa uma nova geração de ferramentas na internet que possibilitam a comunicação, a socialização, a interação, a colaboração, a 
participação, a criação e o compartilhamento de conteúdo. Este recurso proporciona maior interatividade, visibilidade, conteúdos compartilhados e colaborativos, que ocorrem por meio dos SRSO. Os SRSO são oferecidos por instituições privadas denominadas como Redes Sociais Online, que "[...] possuem um conjunto de serviços para auxiliarem os processos de comunicação e de inter-relacionamento de pessoas e de instituições participantes, elaboradas e mantidas por uma ou mais instituições, onde é disponibilizado acesso por meio de ferramentas em web sites ou por aplicativos e oferece acesso a parte dos dados armazenados de seus participantes para coleta por agentes externos" (RODRIGUES; SANT'ANA, 2018, p. 23).

Neste cenário, Carpes (2011) situa as bibliotecas como um locus de encontro nos SRSO, o qual estimula a interação entre os indivíduos da rede, ou seja, a interação social de seus usuários, por meio da disponibilização da informação como etapa da produção do conhecimento. Neste sentido, a biblioteca torna-se um meio institucionalizado que reafirma a ideia de rede como funcionalidade representativa numa sociedade onde a informação passou a exercer um papel central na vida das pessoas. Nessa perspectiva, Castells (2008) afirma que o conhecimento e a informação são fundamentais em todos os tipos de desenvolvimento, já que o processo de produção sempre está vinculado a algum nível de conhecimento e processamento da informação, reflexo de nova forma de desenvolvimento informacional.

Neste contexto, o papel das bibliotecas universitárias deve acompanhar as transformações que ocorrem na sociedade e colaborar para o seu desenvolvimento. Dessa forma, ao se pensar no desenvolvimento sustentável não há como dissociá-lo dos estudos sobre a informação. Sachs (2002) relata que o Desenvolvimento Sustentável possui três pilares fundamentais que são: a relevância social, a prudência ecológica e a viabilidade econômica. Aspectos estes devidamente trabalhados pelos Objetivos do Desenvolvimento Sustentável (ODS), que são o resultado de um acúmulo de experiências, debates e negociações mundiais que foram elaborados em setembro de 2015, dando origem a Agenda 2030. A Agenda 2030 foi elaborada pela Organização das Nações Unidas, a partir do documento "Transformando Nosso Mundo: A Agenda 2030 para o Desenvolvimento Sustentável”, sua vigência é compreendida pelo período de 2016 e 2030, o qual tem como objetivo

Direcionar as políticas governamentais ultranacionais com relação ao desenvolvimento sustentável, por meio de 17 objetivos e de 169 metas que abrangem o desenvolvimento econômico, ambiental e social. A Federação Internacional de Associações e Instituições de Bibliotecas (IFLA) inseriu as bibliotecas na Agenda 2030 por meio da Declaração de Lyon, 
como forma de garantir a atuação das bibliotecas, como agentes promotores do desenvolvimento sustentável. Desta forma, para a IFLA (2015a), as bibliotecas podem contribuir para os 17 objetivos da Agenda 2030, sendo consideradas como instrumentos fundamentais para atuarem em todos os níveis de desenvolvimento social, por meio da democratização do acesso à informação, que associado à disponibilidade de TIC, contribuem expressivamente para o desenvolvimento social e para a melhora da qualidade de vida.

Considerando as problemáticas que permeiam o Brasil, no âmbito do desenvolvimento social e sustentável, mas particularmente o território da Amazônia Brasileira, que abrange os Estados de Roraima, Amazonas, Pará, Amapá, Acre, Rondônia e Tocantins. Pesquisas demonstram apesar dos avanços, os Indicadores de Desenvolvimento Humano (IDH) na Amazônia ainda são inferiores às médias nacionais. Em 2016, o Programa das Nações Unidas para o Desenvolvimento realizou uma lista classificatória (ranking) de Índices de Desenvolvimento Humano Médio (IDHM) dos municípios brasileiros, levando em consideração aspectos relacionados como a renda, longevidade e educação, utilizando dados do ano de 2010 (ATLAS DO DESENVOLVIMENTO HUMANO NO BRASIL, 2013).

Na última pesquisa, o município de Melgaço (Estado do Pará) foi o último colocado com 0,418. A Região Norte apresentou 0,667 e o Estado do Pará 0,646 (PNUD, 2016). Neste sentido, é importante um olhar sobre a Região Amazônica, que é uma região marcada por conflitos e problemáticas a serem resolvidas, seja por meio de políticas públicas ou por outras iniciativas. Destaca-se a contribuição que a Agenda 2030 pode trazer, ao direcionar ações em prol do desenvolvimento sustentável de forma igualitária, a qual poderá contribuir para mudar a realidade dos municípios da Amazônia Brasileira e proporcionar o desenvolvimento social dos habitantes desta região.

Esta pesquisa se concentra no acesso público à informação e considerando o contexto tecnológico da Sociedade em Rede (CASTELLS, 2008), es te estudo traz um recorte para o conteúdo informacional mediado por meio dos Serviços de Redes Sociais Online (SRSO) das bibliotecas analisando se podem trazer uma contribuição para o desenvolvimento social, como prevê a Agenda 2030. Para a realização dessa pesquisa, elegeu-se como universo de pesquisa, a Biblioteca Central Dr. Clodoaldo Beckmann, da Universidade Federal do Pará, por se tratar de uma das maiores bibliotecas universitárias na Região Norte do Brasil e que possui um uso constante de SRSO. A pesquisa, ainda em desenvolvimento, tem o objetivo de analisar qual tipo de conteúdo informacional tem sido mediado por esta biblioteca, mais especificamente no 
perfil institucional do Facebook.

De acordo com os procedimentos metodológicos, está classificada como estudo de caso, utilizando como técnica a observação sistemática e o como instrumento um formulário baseado em estudos prévios e as diretrizes propostas pela IFLA. Os dados foram categorizados quanto ao tipo de conteúdo e as características da mensagem e analisado se o conteúdo informacional mediado possui relação com as ações da IFLA (2015a) que propõe para a colaboração das bibliotecas na Agenda 2030 (IFLA, 2015a). A amostra foi delimitada as postagens realizadas no período dos meses de março a setembro do ano de 2019. Os dados foram categorizados quanto ao tipo de conteúdo postado (campanhas, eventos, avisos/ comunicados/ notícias, divulgação de serviços e informações gerais) e também, quanto às características da mensagem (somente texto; somente imagem; imagem mais links; texto mais links; texto mais imagem; texto mais imagem mais links; vídeo mais texto; vídeo mais texto mais links).

\section{RESULTADOS}

A pesquisa foi iniciada pelo levantamento bibliográfico, posteriormente foram quantificados o número total de postagens, sendo registrado 159 publicações, durante os seis meses de observação, que corresponde ao período entre 15 de março e 15 de setembro de 2019. Os dados coletados por meio das postagens, foram categorizados quanto aos tipos de conteúdo (campanhas, eventos, avisos/ comunicados/ notícias, divulgação de serviços e produtos, informações gerais) e também, quanto às características da mensagem (somente texto; somente imagem; imagem mais links; texto mais links; texto mais imagem mais links; vídeo mais texto e vídeo mais texto mais links). As postagens foram registradas de forma manual, sem a utilização de software para coleta de dados automatizada. A coleta dos dados foi realizada pelos pesquisadores a partir do acompanhamento e monitoramento diário do perfil institucional no SRSO Facebook da Biblioteca Central Dr. Clodoaldo Beckmann, por meio de anotação e categorização dos dados em tabelas.

De acordo com o tipo de conteúdo identificou-se que no mês de agosto foram mediadas mais informações gerais e a divulgação de produtos e serviços, tiveram 5,6\% Campanhas, 16,98\% Eventos, 9,43\% Avisos/ Comunicados/ Notícias, 34,59\% Divulgação de produtos e serviços, 33,33\% Informações gerais. Quanto a característica da mensagem, a média predominou nas categorias texto mais imagem e texto mais imagem mais links, 
quantitativamente, tiveram $1,9 \%$ vídeo mais texto, $1,9 \%$ vídeo mais texto mais links, 11,76\% somente texto, $1,3 \%$ somente imagem, $0 \%$ imagem mais links, $1,3 \%$ texto mais links, $24,8 \%$ texto mais imagem mais links. Observado também as interações com relação as postagens, sendo contabilizado o total de 5.984,263 reações. Os meses que tiveram os maiores índices de reações foram maio (3,697 reações) e julho (2,987 reações). Com relação aos likes, as maiores médias de frequência também permaneceram entre os meses de maio (3,211 likes) e julho (2,488 likes). Levando em consideração as interações, representadas por likes, corações, total de reações são considerados como principal fonte de feed das postagens no SRSO. Pois, apesar da pesquisa não ter como objetivo analisar sobre a interferência dos usuários, faz-se necessário identificar a reação dos integrantes da rede a partir de cada postagem, já que em muitos casos o usuário se comporta como um disseminador do conteúdo postado ou utiliza as postagens originais para interagir. Esse comportamento contribui de maneira significativa para o alcance dos objetivos propostos pela Agenda 2030.

No estudo realizado com a Biblioteca Central Dr. Clodoaldo Beckmann observou-se que esta biblioteca ainda possui uma forte tendência a mediar informações relacionadas a divulgação dos seus produtos e serviços, que segundo a literatura este ainda é o comportamento que tem predominado perante as bibliotecas universitárias brasileira. No entanto, já se percebe uma preocupação desta instituição em mediar informações que possam contribuir para o bem-estar e para a elevação do nível de aprendizagem dos estudantes, por meio da mediação do tipo de conteúdo classificado como informações gerais, que através do quadro "Dicas da Semana" divulga informações quanto ao incentivo à leitura, gerenciamento do tempo de estudo, preservação do acervo, acessibilidade e técnicas de memorização.

Assim como, por meio da tipologia Campanha, em parceria com instituições, como: a Comunidade Cruzeirinho no Baixo Acará, o Hospital Universitário João de Barros Barreto, a Superintendência do Sistema Prisional do Estado do Pará, a Fundação Centro de Hematologia e Hemoterapia do Pará que estão de acordo com as diretrizes estipuladas pela IFLA, contribuindo para o desenvolvimento humano e social da Região Amazônica, corroborando seu papel como uma das maiores bibliotecas universitárias da Região Norte do Brasil. Contribuindo para o alcance dos Objetivos da Agenda 2030 que visa garantir que todos os seres humanos possam desenvolver o seu potencial quanto a dignidade e igualdade, em um ambiente saudável e acessível a todos. 


\section{REFERÊNCIAS BIBLIOGRÁFICAS}

AGUIAR, Giseli Adornato de Aguiar. Uso das ferramentas de redes sociais em bibliotecas universitárias: um estudo exploratório na UNESP, UNICAMP e USP. 2012. $184 \mathrm{f}$. Dissertação (Mestrado em Ciência da Informação) - Universidade de São Paulo, São Paulo, 2012.

ATLAS DO DESENVOLVIMENTO HUMANO NO BRASIL. 2013. Disponível em: http://www.atlasbrasil.org.br/2013.

Acesso em: 30 ago. 2019.

CARPES, Gyance. Percepção de bibliotecários universitários quanto à relevância das redes sociais para o seu cotidiano profissional. 2011. 210 f. Dissertação (Mestrado em Ciência da Informação) - Universidade Federal de Santa Catarina, Florianópolis, 2011.

CASTELLS, M. A sociedade em rede. São Paulo: Paz e Terra, 2008. $630 \mathrm{p}$.

FACEBOOK. BIBLIOTECA CENTRAL UFPA. Disponível em: https://www.facebook.com/bcufpa/?r ef=page_internal. Acessado em: 15 set. 2019

FEDERAÇÃO INTERNACIONAL DE ASSOCIAÇÕES E INSTITUIÇÕES DE

BIBLIOTECAS. As bibliotecas $e$ as implementações da Agenda 2030 da ONU. 2015a. Disponível em: http://archive.ifla.org/III/eb/sustdev02-sp.html. Acesso em: 30 jul. 2019.

FEDERAÇÃO INTERNACIONAL DE ASSOCIAÇÕES E INSTITUIÇÕES DE
BIBLIOTECAS. As bibliotecas e a implementação da Agenda 2030 da ONU. 2015b. Disponível em: https://www.ifla.org/files/assets/hq/to pics/libraries-

development/documents/libraries-un2030-agenda-toolkit-pt.pdf. Acesso em: 31 jul. 2019.

$\begin{array}{llr}\text { PROGRAMA } & \text { DAS } & \text { NAÇÕES } \\ \text { UNIDAS } & \text { PARA } & \text { O }\end{array}$
DESENVOLVIMENTO (BRASIL). Ranking IDMH municípios 2010. 2016. Disponível em: http://www.br.undp.org/content/brazil /pt/home/idh0/rankings/idhm-

municipios-2010.html. Acesso em: 20 de set. 2019.

NAÇÕES UNIDAS NO BRASIL (ONU BR). A ONU e o meio ambiente. 2016. Disponível em: https://nacoesunidas.org/acao/meioambiente. Acesso em: 20 jul. 2019.

NAÇÕES UNIDAS NO BRASIL (ONU BR). A Amazônia e a Agenda 2030 para o desenvolvimento sustentável. [2018?]. 26 p. Disponível em:

https://www.mds.gov.br/webarquivos /Panamazonico/Panamazonico_biblio _port_Amazonia_Agenda2030.pdf.

Acesso em: 30 de ago. 2019.

RODRIGUES, F. DE A.; SANT'ANA, R. C. G. Contextualização de conceitos teóricos no processo de coleta de dados de Redes Sociais Online. Informação \& Tecnologia, v. 5, n. 1, p. 18-36, $2018 . \quad$ DOI: https://doi.org/10.22478/ufpb.23583908.2018v5n1.38077.

SACHS, I. Caminhos para o desenvolvimento sustentável. Rio de Janeiro: Garamond, 2002. 95 p. 
MOTA, K. C. S. L., RODRIGUES, F. A. A Agenda 2030 e o Conteúdo Iinformacional Mediado por Bibliotecas em Serviços de Redes Sociais Online: Um Estudo de Caso para a Biblioteca Central da Universidade Federal do Pará.Complexitas - Rev. Fil. Tem. Belém, v. 4, n. 2, p. 3-11, jul./dec. 2019. Disponível em: http://www.periodicos.ufpa.br/index.php/complexitas/article/view/8050>. Acesso em: 30 de janeiro de 2020. 